\title{
Meningiomas among intracranial neoplasms in Johannesburg, South Africa: prevalence, clinical observations and review of the literature
}

\author{
*Ibebuike $\mathrm{K}^{1}$, Ouma J ${ }^{1}$, Gopal R ${ }^{2}$
}

1. Department of Neurosurgery, Charlotte Maxeke Johannesburg Academic Hospital, Johannesburg, South Africa

2. Department of Neurosurgery, Chris Hani Baragwanath Academic Hospital, Johannesburg, South Africa

\begin{abstract}
Background: Worldwide there are varying reports on the prevalence of meningiomas among intracranial neoplasms. Different reports state intracranial meningiomas, gliomas or metastatic tumours as the most common tumour among intracranial neoplasms. We present our institutions' experience of patients with intracranial neoplasms and a comparison of our findings with those from the literature.

Objective: To determine the relative frequency of intracranial meningiomas among intracranial neoplasms in our environment. Methods: Consecutive patients (151) seen at the CMJAH and CHBAH, Johannesburg, with histologically proven intracranial neoplasms over a 12 month period were analyzed.

Results: The commonest histological types were meningiomas, gliomas and pituitary tumours. Forty eight (31.8\%) patients had histologically confirmed intracranial meningiomas during the study period, $35(23.2 \%)$ had pituitary adenomas and $32(21.2 \%)$ had gliomas. The mean age of the patients was 43 years with a female-to-male ratio of 1.3:1. The peak age range at presentation was in the fifth $(27.1 \%)$ and sixth $(26.5 \%)$ decades.

Conclusion: The study showed that meningioma is the most common tumour among intracranial neoplasms in our environment.

Key words: meningioma, intracranial neoplasm, prevalence, epidemiology

African Health Sciences 2013; 13(1): 118 - 121 http://dx.doi.org/10.4314/ahs.v13i1.16
\end{abstract}

\section{Introduction}

Advances in neuroimaging have improved the diagnosis of intracranial neoplasms. This has made the preoperative diagnosis of intracranial meningiomas almost certain. Reports differ with regards to the prevalence of meningiomas among intracranial neoplasms with some authors reporting meningiomas as having the highest prevalence ${ }^{1,2}$ while others report gliomas ${ }^{3,4}$ or metastatic brain tumours ${ }^{5}$ as the most common tumour among intracranial neoplasms. We therefore looked at the prevalence of meningiomas among intracranial neoplasms in our environment.

We aimed for this study to increase awareness on the prevalence of intracranial neoplasms in our environment while highlighting the local neuroepidemiology of these tumours.

\footnotetext{
*Corresponding author:

Dr Kaunda Ibebuike

Department of Neurosurgery

Wits Medical school, 7 York Road

Parktown 2193, Johannesburg

South Africa

E-mail: ibebuikeke@yahoo.com
}

\section{Methods}

This was a prospective study carried out at the Charlotte Maxeke Johannesburg Academic Hospital (CMJAH) and Chris Hani Baragwanath Academic Hospital (CHBAH), both in Johannesburg, over a period of 12 months.

Approval for the study was obtained from the Human Research Ethics Committee (Medical). Consecutive patients seen at the $\mathrm{CMJAH}$ and $\mathrm{CHBH}$ with histologically proven intracranial neoplasms over a 12 month period were entered into the study.

An investigator-administered questionnaire designed to record details of patient's personal record and clinical data as well as findings from relevant investigations was used. Every new patient was clerked following the order on the pro forma after obtaining informed consent. The patient was then investigated following the standard investigative protocol for intracranial neoplasms including CT scan and or MRI. Following clinical and radiological assessment, treatment was instituted.

All patients underwent neurosurgical operative intervention and all specimens removed at operative intervention were subjected to histology for a final diagnosis. All tumours were brain tumours 
and 11 patients included in the study were considered to have tumour recurrence having undergone previous surgery (predating commencement of the study) for brain tumours and with similar histology to the previous result. The data obtained were analyzed by the use of Epi Info ${ }^{\text {TM }}$ 2002. Data was presented as frequency and percentages.

\section{Results}

During the 12 months study period, 151 patients with intracranial neoplasms were seen in $\mathrm{CMJAH}$ and $\mathrm{CHBAH}$, Johannesburg. Forty eight (31.8\%) patients had histologically confirmed intracranial meningiomas; $35(23.2 \%)$ patients had pituitary adenoma and $32(21.2 \%)$ patients had glioma. The rest of the intracranial tumours seen during the study period including the distribution of recurrent tumours are as shown in table 1.

Table 1: Frequency of distribution of intracranial neoplasms

\begin{tabular}{lcc}
\hline Diagnosis & Frequency & $\mathbf{\%}$ \\
Neoplasms & 48 & $31.8 \%$ \\
Pituitary adenoma & 35 & $23.2 \%$ \\
Glioma & 32 & $21.2 \%$ \\
Metastatic tumour & 9 & $6.0 \%$ \\
Craniopharyngioma & 4 & $2.6 \%$ \\
Ependymoma & 4 & $2.6 \%$ \\
Medulloblastoma & 4 & $2.6 \%$ \\
Schwannoma & 3 & $2.0 \%$ \\
Myeloma & 3 & $2.0 \%$ \\
Lymphoma & 2 & $1.3 \%$ \\
Epidermoid cyst & 2 & $1.3 \%$ \\
PCNSL & 1 & $0.7 \%$ \\
Meningeal haemangiopericytoma & 1 & $0.7 \%$ \\
Haemangioblastoma & 1 & $0.7 \%$ \\
Atypical teratoid/rhabdoid tumour & 1 & $0.7 \%$ \\
PNET & 1 & $0.7 \%$ \\
Total & 151 & $100 \%$ \\
\hline Tumor Recurrence & & \\
Pituitary adenoma & 6 & $54.5 \%$ \\
Glioma & 4 & $36.4 \%$ \\
Meningioma & 1 & $9.1 \%$ \\
Total & 11 & $100 \%$ \\
\hline
\end{tabular}

Eighty four $(55.6 \%)$ patients were females and 67 $(44.4 \%)$ were males. The mean (range) age of patients with intracranial neoplasms was 43 (2-73) years with a median age of 46 years. The mean (range) age for male patients was 41.2 (2-73) years while that of female patients was 44.4 (2-69) years.
The peak age range was in the age group 41-50 years and 51-60 years accounting for 27.1\% and $26.5 \%$ of patients and corresponding to the fifth and sixth decades of life respectively. As shown in table 2, females accounted for higher prevalence of brain tumours with aging whereas males accounted for higher prevalence in childhood and younger age.

Table 2: Age and gender of patients at presentation

\begin{tabular}{|c|c|c|c|c|}
\hline \multirow{2}{*}{$\begin{array}{l}\text { Variable } \\
\text { Gender }\end{array}$} & \multicolumn{3}{|c|}{ Number of patients } & \multirow[t]{2}{*}{$\%$} \\
\hline & & & & \\
\hline Male & \multicolumn{3}{|l|}{67} & $44.4 \%$ \\
\hline Female & \multicolumn{3}{|l|}{84} & $55.6 \%$ \\
\hline Total & \multicolumn{3}{|l|}{151} & $100 \%$ \\
\hline $\begin{array}{l}\text { Age } \\
\text { group } \\
\text { (years) }\end{array}$ & Males & Fems & s Total & \\
\hline $1-10$ & 5 & 4 & 9 & $6.0 \%$ \\
\hline $11-20$ & 5 & 3 & 8 & $5.3 \%$ \\
\hline $21-30$ & 8 & 5 & 13 & $8.6 \%$ \\
\hline $31-40$ & 13 & 13 & 26 & $17.2 \%$ \\
\hline $41-50$ & 13 & 28 & 41 & $27.1 \%$ \\
\hline $51-60$ & 15 & 25 & 40 & $26.5 \%$ \\
\hline $61-70$ & 7 & 6 & 13 & $8.6 \%$ \\
\hline $71-80$ & 1 & 0 & 1 & $0.7 \%$ \\
\hline Total & 67 & 84 & 151 & $100 \%$ \\
\hline
\end{tabular}

\section{Discussion}

Meningiomas are estimated to constitute between $13-26 \%$ of primary intracranial neoplasms, ${ }^{6}$ although early combined results from several large hospitalbased brain tumour studies showed that the incidence of meningiomas is approximately $20 \%$ of all intracranial tumours. ${ }^{7,8}$ However, the recent report of the Central Brain Tumor Registry of the United States shows consistently high incidence of meningiomas, accounting for $35 \%$ of all brain tumors diagnosed in the United States ${ }^{9}$ in 2004-2008.

In this study meningiomas accounted for $31.8 \%$ of intracranial neoplasms and were the most common tumour among intracranial neoplasms in our environment. They were closely followed by pituitary adenomas, giomas and metastatic tumours in that order. The high prevalence of meningiomas in our study compares with recent studies by Idowu et $\mathrm{al}^{10}$ in Ibadan (35\%), Idowu and Apemiye ${ }^{11}$ in Lagos (30\%) and the recent report of the Central Brain Tumor Registry of the United States $(35 \%)^{9}$ which all reported meningiomas as the most 
common brain tumour in their series. Our findings also compare with the study by Das et $\mathrm{al}^{3}$ in Singapore $(35.1 \%)$ in which meningiomas ranked highest among symptomatic brain tumours in their environment. Although a low incidence of intracranial tumours has been reported among the Japanese, in a recent study by Nakamura et $\mathrm{al}^{12}$ meningiomas were the most common tumour. However, our findings contrast with other reports which showed that gliomas were the most common tumour among intracranial neoplasms. ${ }^{13,14,15}$

In a recent study in Osaka, Japan by Nomura et $\mathrm{al}^{16}$ the last 10 year period from 19952004 of their 30 year study from 1975-2004 showed that the age-standardized incidence rates of meningioma decreased significantly, but those of glioblastoma did not. However, they advised caution in accepting their results due to several limitations, one of which was the likelihood of under-reporting benign tumours. Still, there are a few old studies relating to the higher prevalence of gliomas among intracranial neoplasms when compared to that for intracranial meningiomas which has more recent studies indicating its higher prevalence. Christensen et al, ${ }^{17}$ in a 55 year study in Denmark reported 3.9 fold increase in the incidence of meningiomas from 1943 to 1997. In contrast, the incidence of gliomas increased by only a 1.7 fold during the same period. They noted in their study that improved diagnostic modalities have reached their maximum for gliomas. None the less the same was not observed for meningiomas, and this may suggest that meningiomas were under-reported until recently.

The low prevalence of recurrent meningiomas may reflect the short duration of the study since these tumours are slow growing, although it may also reflect the propensity for cure with total tumour resection as most tumours are histologically benign. ${ }^{18}$ However, even with total tumour resection and the benign nature of these tumours, the recurrence rates for intracranial meningiomas range from $10-20 \%{ }^{18}$ Metastatic brain tumour which has also been reported to be the most common type of tumour in the brain ${ }^{5,15}$ had a very low prevalence in our study. The low prevalence for metastatic tumours in our study may be a reflection of the observation in most reports that resection of metastatic brain tumour is generally reserved for patients with stable systemic disease who are in good neurologic condition. ${ }^{19}$ The benefit of histologic confirmation in most cases of metastatic brain tumours is therefore missed.
The findings in this present study showed that intracranial meningiomas are the most prevalent among intracranial neoplasms in our environment and this is consistent with reports which indicate that the incidence of intracranial meningiomas is high in Africans. $^{20}$

\section{Conclusion}

Although our findings may indicate a high trend in the prevalence of intracranial meningiomas in our environment, it may also be the result of the improved detection rate through the increasing availability of modern diagnostic neuroimaging facilities in our hospitals such as CT and MRI scans. A larger multicentre and longer duration study in different cities in South Africa may help to have a clearer picture of the prevalence of this tumour in our environment.

\section{References}

1. Campbell BA, Jhamb A, Maguire JA, et al. Meningiomas in 2009: Controversies and Future Challenges. A J Clin Oncol 2009;32:73-85.

2. Wiemels J, Wrensch M, Claus EB. Epidemiology and etiology of meningioma. J Neurooncol 2010;99:307-314.

3. Das A, Chapman CAT, Yap WM. Histological subtypes of symptomatic central nervous system tumours in Singapore. I Neurol Neurosurg Psychiatry 2000;68:372-374.

4. Grant R. Overview: brain tumour diagnosis and management/Royal College of Physicians guidelines. J Neurol Neurosurg Psychiatry 2004;75:18-23.

5. Davis CP. Brain Cancer. emedicinehealth http:/ /www.emedicinehealth.com/brain_cancer/ article_em.htm. (Accessed 28 Nov, 2011).

6. Hashiba T, Hashimoto N, Izumoto S, et al. Serial volumetric assessment of the natural history and growth pattern of incidentally discovered meningiomas. J Neurosurg 2009;110:675-684.

7. Longstreth WJJ, Dennis LK, McGuire VM, et al. Epidemiology of Intracranial Meningioma. Cancer 1993;72:639-648.

8. Bondy ML, Ligon BL. Epidemiology and aetiology of intracranial meningiomas: a review. J Neurooncol 1996;29:197-205.

9. Central Brain Tumour Registry of the United States (CBTRUS) website. http:// www.cbtrus.org/2012-NPCR-SEER/ CBTRUS_Report_2004-2008_3-23 PubMed 2012.pdf. (Accessed 28 May, 2012). 
10. Idowu O, Akang EEU, Malomo A. Symptomatic Primary Intracranial neoplasms in Nigeria, West Africa. JNS Turkish 2007;24:212218.

11. Idowu OE, Apemiye RA. Delay in presentation and diagnosis of adult primary intracranial neoplasms in a tropical teaching hospital: A pilot study. IJS 2009;7:396-398.

12. Nakamura H, Makino K, Yano S, Kuratsu J, Kumamoto Brain Tumor Research Group. Epidemiological study of primary intracranial tumors: a regional survey in Kumamoto prefecture in southern Japan-20-year study. Int $J$ of Clin Oncol 2011;16:314-321.

13. Dobec-Meic' B, Pikija S, Cvetko D, et al. Intracranial tumors in adult population of the Varazdin County (Croatia) 1996-2004: a population-based retrospective incidence study. J Neurooncol 2006;78:303-310.

14. Lona C, Tabiadon G, Curro DB, et al. Incidence of primary intracranial tumors in the province of Bolzano 1980-84. Ital J Neurol Sci 1988;9:237241.
15. Campos S, Davey P, Hird A, et al. Brain metastasis from an unknown primary, or primary brain tumour? A diagnostic dilemma. Curr Oncol 2009;16:62-66.

16. Nomura E, Ioka A, Tsukuma H. Trends in the incidence of primary intracranial tumors in Osaka, Japan. Jpn J Clin Oncol 2011;41:291-294.

17. Christensen HC, Kosteljanetz M, Johansen C. Incidence of gliomas and meningiomas in Denmark, 1943 to 1997. Neurosurgery 2003;52:1327-1333.

18. Gelabert-González M, Serramito-García R. [Intracranial meningiomas: I. Epidemiology, aetiology, pathogenesis and prognostic factors]. Rev Neurol 2011;53:165-172.

19. Nussbaum ES, Djalilian HR, Cho KH, et al. Brain metastases: Histology, multiplicity, surgery, and survival. Cancer 1996;78:1781-1788.

20. Haddad G. Meningioma. eMedicine Nov 3, 2011 http://emedicine.medscape.com/article/ 1156552-overview. (accessed 26 Nov, 2011). 